\title{
INFLUENCING FOLLOWERSHIP: UNDERSTANDING THE PERSPECTIVE OF THOSE LEADING ACTIVE DISCUSSIONS ON QUORA
}

\author{
Martin C. Nwadiugwu \\ University of Nebraska at Omaha, 2626 PKI, University of Nebraska Omaha, Omaha, NE 68182, USA
}

\begin{abstract}
As social media influence becomes popular, having an understanding of why some posts are more highly followed than others as viewed from the perspective of those leading the post allows us to gain insight on how followership is being influenced. A qualitative study of eight participants leading active discussions on Quora was conducted using semi-structured in-depth interviews, followed by thematic analysis. The open coding method was used to iteratively code related answers to develop themes. Results suggest that copyright tactics, controversial answers and sharing new information are some of the mechanisms for influencing followership. These mechanisms are built overtime through strong engagement and self-awareness by writing a consistently well-thought-out answer. The motivation for leading and writing answers on Quora were more intrinsic than extrinsic. Most participants believed influencing followership should not be a concern if one has the right message.
\end{abstract}

\section{KEYWORDS}

Followership, Influence, Leadership, Online Communities

\section{INTRODUCTION}

Quora is a social media online platform that brings together people with diverse perspective to understand each other in a culture of knowledge sharing (Quora 2020). It is a popular question and answer site that differs from others because it integrates social network into its basic structure (Maity et al. 2017; Wang et al. 2013) and a platform where users write and answer questions; the best answers are seemingly identified by upvotes and downvotes (Patil and Lee 2016). It has been previously reported that Quora and draws about 200 million visitors monthly and over 13 million questions have been asked as at 2017 (D'Angelo 2017; Quora 2017). Interestingly, while there are many questions and answers on the platform, it is not widely known why some answers are more influential than others (Paul et al. 2012). It is pertinent to understand the experiences and perspectives of those writing answers and leading discussions on Quora to gain understanding into how followership is being influenced.

Assessment of Quora shows it follows the principles for online community design as suggested by Kim (2003). The platform has a defined purpose that understands the intended users and services to be delivered in the "Why Quora Exist" section of the home page that also highlights the mission statement of the community which simply is: "to share and grow the world's knowledge" (Quora 2020). An overview of the site content is shown in a simple and usable way for visitors allowing them to navigate the design of member profiles that evolve over time, and to be made aware of their rights and responsibilities in the community that is seen in the "conduct policy" link at the bottom of the home page. While there is a clear code of conduct, controversy, debate and allowing members to be different in a way that is not disruptive can be seen in the online community and this creates the fun (Kim 2003). Ranking of members and level of completed membership profile is also visible to the user so that more accurate information can be updated. As a Q\&A online platform, discussion areas exist engaging the community with effective leadership (Kim 2003). New members are allowed to lead discussions and answer questions which is in line with the principles of creating a balance that accommodates new members without alienating those that are active (Kim 2003). The focus of this study is on effective leadership within discussion areas on Quora. It aims to gain insight into understanding why some answers are highly followed; specifically, to understand if those leading active discussions are self-aware and if they play a role in influencing followership, comments and upvotes. The 
social mechanisms and decision-making processes that help them judge if an answer would be trending is a focus of the study. For example, 1) What do social media influencers hope to gain from writing answers or leading a discussion? 2) What influences active discussion on Quora? In other words, which scenarios will encourage a post to trend and 3) how does the topic affect user experience and followership on Quora?

A closer observation of the platform showed that each user has a profile history that keeps track of all answers and questions an individual had posted, the number of shares and followers they have. The Technology space within Quora (observed from February 6 to March 15 2020) was selected for the study and the activities of people who had posted answers that consistently received considerable activity with about 50 or more comments, upvotes and shares were observed. While some users on the platform could be identified with pseudonyms, only those who used supposedly real identities were further observed and selected as potential participants because real identities build perception of trust for high quality answers (Paul et. al 2012; Kollock 1998). Although popularity based on real identities may be biased due to the fact that users may in general have an attachment and preferential treatment to some notoriety which can influence the voting and promotion of answers that may not be novel (Barabási \& Albert 1999); the social voting is still considered as a way of identifying quality answers (Paul et. al 2012) and was used in this study as the criteria for selecting participants leading active discussions on Quora.

The remaining sections of the study will highlight the purpose and the methodology used. Next, a weaving of the in-depth interview from the participants will be synthesized to answer the study question of how followership is being influenced. Answers to questions about the participant's understanding of leadership, motives for leading online discussions, self-awareness of user behavior, cherry-picking discussion topics, mechanisms for influencing followership and how unrelated questions affect user experience will be sought. The study will conclude with a summary of the narration and how users of online communities may benefit from future design improvements.

\subsection{Purpose of the Study}

Although every member in an online community can exhibit leadership behavior, some are more effective in influencing others (Zhu et al. 2012). According to Weeks et al. (2017) opinion leaders and highly active users in online communities can be influential in persuading their peers and believe they are both indirectly and directly able to persuade others. In a study on the effectiveness of shared leadership in online communities, Zhu et al. (2012) found that person-focused leaders are more effective in influencing others to contribute, while legitimate leaders are the more influential. However, leadership can only exist when there is a followership, and the advent of the internet has given rise to global followership allowing leaders and followers to be united in a partnering process (Tolstikov-Mast and Yulia. 2016). It is therefore important to know the mechanisms that influences followership and why some answers and discussion posts in online communities have more participation than others especially given the theory that leadership is founded on the desire for control over others (Ford and Harding 2018).

\section{METHODOLOGY}

The study was conducted using a semi-structured, in-depth interview. Eight participants were selected for the study comprising of six males and two females as seen in Table 1. All participants were above 18 years of age. A pilot test ensured adequate refinement of the interview questions according to the interview guide (Creswell 2007). The interviews were conducted via Zoom and were audio-recorded except for one that was text-based. The interviews lasted from 15 to 30 minutes.

Table 1. Characteristics of Selected Participant

\begin{tabular}{|l|l|l|l|l|l|l|}
\hline Participants & Gender & Country & Followers & Answers & Content views & Job Highlight \\
\hline P01 & Male & USA & 245 & 129 & 932,400 & College Student \\
\hline P02 & Male & USA & 510 & 120 & 807300 & Online Writer \\
\hline P02 & Male & Nigeria & 504 & 3923 & $7,600,000$ & Blogger \\
\hline P04 & Female & USA & 4310 & 2787 & $5,600,000$ & Patreon Analyst \\
\hline P05 & Male & USA & 1075 & 4436 & $13,300,000$ & Business Adviser/Writer \\
\hline P06 & Male & France & 74532 & 387 & $29,600,000$ & Stand-up Comedian \\
\hline P07 & Male & USA & 6558 & 566 & $7,100,000$ & College Student \\
\hline P08 & Female & USA & 3828 & 319 & $2,900,000$ & Borderline Advocate \\
\hline
\end{tabular}


The terms of confidentiality were addressed and the participants gave recorded consent to use the transcript of the interview in the study. Participant names were de-identified as seen in Table 1. This is in line with suggestions that pseudonyms, and disguising person names are recommended for protecting confidentiality of research subjects (Bruckman 2006). The participants were sent the initial transcript at the end of the interview to allow them make clarifications on possible errors in the transcription process. The following open-ended questions were asked:

1. Tell me about your understanding of being a leader on Quora?

2. What do you hope to gain from leading a discussion on Quora?

3. Tell me about any concern you have in leading an online discussion?

4. What scenarios will make a post trending on Quora?

5. Do people look up to suggestions on Quora in making their decisions?

6. How do unrelated questions affect user experience/behavior?

7. Anything else you would like to share?

The data was analyzed using was a thematic methodology that incorporated an inductive approach to generate explanations from the data (IER 2014). The rationale for using this method was because the aim of the interview follows a strategy that seeks an explanation of a substantive theory (IER 2014) and the thematic analysis provides a well-structured approach in explaining the data (Nowell et al. 2017).

\subsection{Participants Interview}

Table 2 shows the thematic analytic approach used to code the interview transcripts and to iteratively develop themes related to the interview questions as suggested by Strauss and Corbin (1990) and Vollstedt and Rezat (2019)

Table 2. Coding Interview Data

\begin{tabular}{|c|c|}
\hline Interview Extract & Codes \\
\hline $\begin{array}{l}\text { "I would categorize being a leader as one who has lots of followership" - P01 } \\
\text { "I have been influenced by post made on Quora." - P03 } \\
\text { "I write and interact a lot but don't think of myself as a special leader" - P04 } \\
\text { "Some people are more successful than others, it depends on topics they write" - P05 } \\
\text { "you can accrue a following based on merit" - P06 } \\
\text { "a person with a lot of followers on Quora" - P07 } \\
\text { "I am diagnosed with Borderline Personality Disorder, I created my own page. When Quora introduced } \\
\text { spaces, I was invited to be an admin for three pages" - P08 }\end{array}$ & $\begin{array}{l}\text { Followership as } \\
\text { a criteria for } \\
\text { influential } \\
\text { leadership }\end{array}$ \\
\hline $\begin{array}{l}\text { "People on Quora take a little bit more time to write answers unlike in Reddit"- P01 } \\
\text { "I never seek it out." - P02 } \\
\text { "I try to have well founded argument and express my bias" - P04 } \\
\text { "After you've established yourself.. you can broadcast your own message "-P05 } \\
\text { "do something commercial do... sell your product"-P06 } \\
\text { "start first by giving answers that people enjoy and getting their attention."-P07 }\end{array}$ & $\begin{array}{l}\text { Followership is } \\
\text { built not made }\end{array}$ \\
\hline $\begin{array}{l}\text { "a post I made surprisingly ended out getting sent to about two million people" - P01 } \\
\text { "I sometimes post funny answers to create humor for those reading my post"-P03 } \\
\text { "I would not write answers that are inimical to societal values or on things"-P05 } \\
\text { "I use copywriting tactics to make my answers...there are topics I wouldn't lead"- P06 } \\
\text { "First, you have to capture the audience's attention,... with a controversial post" P07 } \\
\text { "I started writing to see if my understanding of my disorder was accurate."- P08 }\end{array}$ & $\begin{array}{l}\text { Leaders cherry- } \\
\text { pick topics }\end{array}$ \\
\hline $\begin{array}{l}\text { "One answer could be seen by a million people and another seen by five."-P01 } \\
\text { "You have to respect your readers and not to give them an error-filled answers" - P02" } \\
\text { "Quora audience is more reflective of people on the internet and like Tiktok"- P06 } \\
\text { "After you capture their attention, you keep it by writing interesting answers"- P07 }\end{array}$ & $\begin{array}{l}\text { Self-awareness } \\
\text { of user behavior }\end{array}$ \\
\hline $\begin{array}{l}\text { "to just share what I knew for whoever would listen".-P01 } \\
\text { "I do not want to gain anything. I enjoy answering questions"- P02 } \\
\text { "no monetary motives.. to seek approval with up-votes and top-writer ranks"-P03 } \\
\text { "I have a patreon (crowdfund) - I actually get paid by people to write on Quora"-P04 } \\
\text { "for knowledge and sharing your experience" - P05 } \\
\text { "I hope to connect with other people on Quora"-P06 } \\
\text { "I try to influence public perception about overlooked topics I care about"-P07 } \\
\text { "is to remove the stigma that is attached to my disorder."-P08 }\end{array}$ & $\begin{array}{l}\text { Motivation } \\
\text { leading }\end{array}$ \\
\hline
\end{tabular}




\subsubsection{Followership is the Criteria for Determining Influential Leadership}

Studies have posited that the experience of leadership roles varies in relation to how followers differ in acting out their roles (Carsten et al. 2017). Followership role orientation co-produces leadership outcome of influence, motivation, and perceived follower support (Carsten et al. 2017). In other words, influential leadership is hinged on the perception of follower's support which motivates the leader. The ability to influence participation on Quora was highlighted by 7 participants as a feature of leadership. One participant said: "I would agree and categorize being a leader as one who has lots of followership. I also think being a leader is being one with integrity and that's what is kind of falls down to"-P01. Apart from having a lot of followership, the ability to have strong engagement in a culture of sharing was considered a criterion for leading and six participants alluded to this. For example, a participant mentioned: "In Quora there are a lot of people who would post [comment on a post] but won't write a post. The silent majority are probably people who read and don't interact at all"- P04. There was an agreement that most users on Quora were passive rather than active which necessitate that discussion leaders would strategize for a stronger participation. Moreover, user participation is vital to sustain the platform as a "third place" for Q\&A where regular discussion leaders makes it lively without which it would be an empty space (Oldenberg 1999).

The focus topic was suggested as the reason for the differences and success of influential people leading discussion post on Quora. According to the fifth participant: "Everybody is different on Quora with different perspectives. Some people are more successful than others and it depends on the topics they write about. Some topics I have written about got only about 500 views. After you've established yourself in some select topics that is more followed, you can broadcast your own message very effectively" The focus topic is usually what the participant cares about. Two participants agreed that taking up discussion leader roles on topics have attracted some attention; one of them stated: "I am diagnosed with Borderline Personality Disorder. I started writing to see if my own understanding of my disorder was accurate. I have no ability to perceive myself. It's part of the disorder. I have no identity. We can, without treatment, become emotional remoras. What I discovered was this...to show what I write makes sense to many people. And my voice has a way of healing or helping others on their journey. I created my own page, Invisible Lines, mostly to blog my day to day frustrations. My own page did not take off, but when Quora introduced spaces, I was invited to be an admin for three pages."- P08. The number of followers an individual has is able to determine their influence on Quora, and because the Ask-to-Answer design of the platform allows users to tag other Quora members, highly active users are promoted as they would be tagged in most questions increasing their influence (Paul et al. 2012). Five participants alluded to this and one particularly stated: "I try to influence public perception about topics I care about that are being overlooked and people don't have much information about it. I try to give my answers, make them popular so some people will learn about it for the first time and others who know about it will have complete information."-P07.

\subsubsection{Followership is Built not Made}

The process of influencing followership and participation on Quora requires expertise and experience that builds up overtime. The fifth participant explicitly revealed that this building process is shrouded in mystery: "Sometimes you write answers that don't get any readership. I have noticed that if you focus on what you really know and what you can help people with, eventually your articles would start getting attention. This is very hard in the beginning. When I started on Quora and wrote an answer, I would be lucky if 100 or 200 people read it. Everybody that seems to be doing well on Quora in terms of readership, tend to write in the same subject and have a good broad knowledge and a certain message in the way they write." One way Quora can support newcomers sharing great ideas and leading discussions to increase their audience base could be by making their feeds visible in order to encourage other members pay more attention to them (Burke et al. 2009).

Leadership is built on the theory of followership, and leaders have been reported to compare followers to their ideal expectation of followership, and judge them against this model sometimes without considering the actual behavior of the follower (Ford and Harding 2018). Determining what scenarios would make a post trending on Quora, five participants agreed that this process takes time; P07 stated: "First, you have to capture the audience's attention, let's say If you have an intriguing picture or a controversial post or something like the corona virus. After you capture their attention, you have to keep it by writing answers that are interesting and easy to understand. Popularity of answers on Quora boils down to talking about things that are interesting where you have a unique perspective that keeps the audience's attention." While this may be true, some people are necessarily convinced that you don't need to worry about followership if you have the right motivation as the fourth participant posited: "I don't worry about trending, but on writing good stuff and 
that is the reward. My post on coronavirus is just an example. Sharing answers on topics that have not been shared is a motivation. On Quora, I can trust the audience to choose what they want and don't want to absorb. I am very widely read and that is what makes my post trending."

\subsubsection{Leaders of Online Discussion Cherry-Pick Topics}

People in active leadership roles could improve the relationship with followers by advancing connections that elevates, stimulates, and transform both followers and leaders into confident leaders and moral agents respectively (Burns 1978). This perhaps could be the rationale why active leaders of a discussion post in online communities cherry-pick their topics to influence conversation. Answering the question on what will make a post trending on Quora the sixth participant stated: "I use copywriting tactics to make my answers. I post about sex usually on first headline, because people are naturally gravitating towards that. Things like sex, racism, and something controversial generally elicits more comments. Although I would not like to do those things, sometimes when I have written answers of great value, they got almost no traction. Sometimes you have to do something commercials do such as the use of sex, cars to sell your product and then once they are inside you give better forms of advice." Another participant [P03] was also in agreement saying: "You want to make sure your answers are flawless and free of errors when you lead a discussion post... when people see that your answers are impeccable, they would know you are competent and want to follow you." On the other hand, there were other contrasting views; participant one and two mentioned not seeking to influence followership, the first participant who was particularly surprised at the traction gotten by a recent post said: "I have been surprised a couple of times. There was a post I made that was a little bit technical and I did not imagine it would elicit much interest. It ended out getting sent to about two million people because they have an email where they broadcast messages to people, and it was a little bit surprising, I really don't know what causes them to be successful". An overview of the answers given showed that six participants alluded to the fact that what made an answer on Quora trending involved a strategic process and that if one is an expert in a range of topic and had a wealth of experience on advising, they would not be concerned with scenarios that makes a post trending. This agrees with the study by Meindl (1995) who stated that charisma is influenced by discussions among followers and it arises from the minds of followers who concludes that a leader is charismatic.

\subsubsection{Leaders of Online Discussion are Self-Aware of User Behavior}

Emerging studies in leadership have been focused on followership that assumes that there is a common purpose for both leaders and followers and a mutually beneficial relationship (Shamir et al. 2007; Challef 1998; Ciulla 1998). Those who lead discussions and write answers on Quora are self-aware of their audience. A participant [P03] on being asked for additional comment on experience writing answers on Quora mentioned: "Being a writer means that you are sometimes "naked" [revealing secrets] just in a bid to convince people that you are sure of what you are talking about. Many secrets about countries, government and people are being exposed in a bid to get up-votes." Apart from knowing their audience, they are also aware of the designs of online communities and how they affect user behavior. A participant [P05] said: "In standard media you could get one answer, one article, one perspective unlike in Quora where you could get fifty articles if it's a popular question. Also, there are people on Quora who have not written anything but come there to get knowledge." Participant six also shared similar opinion: "I think one of the good things about Quora is that you could get answers to a question you really didn't know you needed an answer to. I think that the randomness of that makes the user experience more exciting because for example, a big problem with Instagram which is a completely controlled platform [i.e. you are not seeing anything except things you want to see] therefore you are getting a very myopic sense of the world and there is a level of non-randomness which gets boring and stale. The Quora stream is more similar to Tiktok where you get what you want. Those algorithms tend to heighten user experience".

Accordingly, there are similarities in the opinion of the participants that influential leaders of online discussion write answers using tactics that increases participation because they are aware of their audience, their content and the design of the online community. This self-awareness makes them reconsider feedback from their readers to keep the followership they have been able to acquire overtime. The eight-participant said: "For a while I used to write some trashy answers. I used to write answers with underlined italics to capture attention, but people were of the opinion that "this isn't cool" and that it looks like am hunting for attention and not providing as much value as I could, which made me rethink how am writing. Yes, I will change it if people point how badly am writing." 


\subsubsection{The Motivation for Leading is Mixed}

According to Mamykina (2011) intrinsic rewards such as the desire for learning, altruism or extrinsic benefits such as social or material rewards have been highlighted as the motivations for participating in online question and answer sites. Some sites such as YouTube have a reward mechanism for active users with rich content, while other have point based reward system (Mamykina 2011). However, Quora is devoid of point-based reward system (Paul et al. 2012). Therefore, to understand the intrinsic or extrinsic motivations for leading discussions, participants were asked what they hoped to gain from leading a discussion on Quora. Seven out of eight participants mentioned intrinsic rewards such as altruistic actions (helping people with advice, direction, spreading knowledge), social interaction and building social capital as the main motivations. Providing further information on the motivations for writing answers on Quora [P03] stated: "Some people have come to Nigeria to meet me and learn more about the country and what I have written. Some of my followers who are in a dating relationship with a Nigerian call me to know about the country and verify the authenticity of the locations and its safety. Quora is a place where people build relationship and trust one another based on the answers and suggestions they are reading. It is one of the most visited sites in the world and I think people take post there seriously."

In addition, others have seized the networking opportunity provided by the online platform to create awareness to remove stigma attached to a disorder. P08 said: "My primary goal for writing on Quora is to remove the stigma that is attached to my disorder. Borderlines have a particularly brutal stigma attached to their disease. A whole lot of support are subjective and very few facts. Clinicians hate to treat us. We burn through people and their emotions like a woodchopper and it takes years of multidisciplined help to get anywhere near better. I have done a lot of work but still more to do. I also managed to create a community of both Borderlines, and former partners, who are willing to put aside their anger to get to a place of acceptance, radical acceptance. It feels very validating, that I have accomplished through real hard work, the ability to bridge the communication gap that is so frustrating about BPD." Furthermore, social appraisal is the popular reward mechanism that exists on Quora, and while some participants have leveraged on their popularity on Quora for extrinsic benefit from external sources, others have called on the designers of Quora to recognize and reward creative contributions. P06 said: "I think that Quora should add 'tip features' where people can give creators money. There is an immense amount of value being created on the internet, and the people that are creating it for the first time in human history are not getting access to profit from it. Am not saying that platforms should pay people, but, if someone writes an answer that changes your life [like benefits from YouTube] we can create a line between consumer of their content and the creators bank account. I think with this, we would get a better content; and this is the reason why YouTube has better videos than a lot of NetFlix documentaries."

The motivation to offer these services is aided by the design of Quora platform. It was suggested that the design algorithms on Quora frequently engineers related notifications to a user as unrelated feeds would discourage participation. On how an unrelated post would affect the user experience the seventh participant stated: "I think Quora does a good job with its algorithms about giving you things you would be interested in. At some point I once interacted with a topic (conjoined twins) that I cared very little about but I enjoyed it and kept on getting questions and answers [scientific] about it and there's no way for me to indicate that am uninterested in it. And this causes more unrelated things to pop up in my feed and notification which drives me away from Quora."

\section{DISCUSSION/CONCLUSION}

An in-depth study to understand the perspectives of those writing answers and leading discussions on Quora was conducted to comprehend mechanisms that influences comments, posts or suggestions and how discussion topics affect user experience and followership on Quora. It was found that the motivation to lead discussions were mostly intrinsic (altruism, knowledge sharing) than extrinsic (material reward) suggesting that providing balanced argument and reducing bias is highly valuable. The mechanisms that influences followership and participation in a discussion requires expertise and experience and this happens over time. It includes the ability to engage strongly and to improve relationship with followers by advancing connections that excites both parties (Burn 1978). This greater knowledge of deliberative etiquette is needed to resist targeted incivility (Gervais 2015). A well-thought-out answer were largely considered as a means of reaching a targeted audience and increasing participation, while biased and error-prone messages could lead to distrust. It can be suggested from the study that those who lead discussions and write answers on Quora are 
self-aware of their audience; and perhaps use tactics that increases participation. Copyright tactics, controversial answers and sharing new information were some of the highlighted mechanisms for influencing followership.

Exploring the concept of building leadership on the theory of followership, most participants were of the opinion that followership should not be a concern if one has the right message. However, it has been reported that leaders have an ideal expectation of followership that differs from reality (Ford and Harding 2018). The study by Paul et al. (2012) to understand reputation mechanisms on Quora found that influential users get more votes than are deserving for their answers. This is further supported by Lerman and Galstyan (2008) who studying social voting on Digg noticed that some users on the site had their topics promoted in the front page of the platform because of their notoriety in large social networking sites. Although it has been reported that Quora uses an algorithm called PeopleRank to rank answers based on some select criteria, it has also been suggested that this could potentially lead to undue influence because it highly ranks the answers of popular users even when their answers are not catchy (Paul et al. 2012). This study suggests that active users influences follower perception, and the platform design of Quora promotes this influence by allowing users tag other members to a particular answer.

In summary, findings from the study provides important insight on the mechanisms influencing followership. This information would be paramount in understanding the design implications for knowledge-building in online communities. Further studies may be carried out to investigate preferential attachments that influences social voting from the perspective of the follower. Also, understanding the way information is produced and circulated on Quora in relation to the PeopleRank algorithm is another interesting area for future studies.

\section{COMPLIANCE WITH ETHICAL STATEMENTS}

(a) Ethical Approval: This article was approved by the IRB

(b) Funding: The author declares no research funding for this study.

(c) Conflict of Interest: The author declares no conflict of interest

(d) Availability of Data and Material: Tables and data used are duly provided.

(e) Authors' Contribution: M.N. developed the study question, designed the research and typed the manuscript.

\section{ACKNOWLEDGEMENT}

I would like to acknowledge Briana Morrison (PhD), Assistant Professor, University of Nebraska at Omaha for reviewing the manuscript.

\section{REFERENCES}

Barabási, A.-L.; R. Albert. Emergence of scaling in random networks" Science 286, 5439 (1999), 509-512.

Burke, M., Lento, T. and Marlow, C. (2009) Feed Me: Motivating Newcomer Contribution in Social Network Sites. http://www.thoughtcrumbs.com/publications/paper0778-burke.pdf [Accessed: January 16 2020]

Burns J. 1978. Leadership. New York, NY: Harper and Row.

Bruckman, A. (2006) Teaching students to study online communities ethically. Journal of Information Ethics, 3(11), p.82-95.

Carsten, M. K., Uhl-Bien, M. and Huang, L. (2018) 'Leader perceptions and motivation as outcomes of followership role orientation and behavior'. Leadership, 14(6), pp. 731-756. doi: 10.1177/1742715017720306.

Challeff, I (1998) The Courageous Follower: Standing Up To and For Our Leaders, San Francisco: Berrett-Koehler Pub.

Ciulla, J (1998) Ethics: The Heart of Leadership, London: Praeger.

Creswell, J.W. (2007) Qualitative Inquiry and Research Design: Choosing Among Five Approaches: International Student Edition. Thousand Oaks, California: Sage Publications.

D'Angelo, A._(2017) How many people use Quora. Available: https://www.quora.com/How-many-people-use-Quora-3 
Ford, J., Harding, N. (2018) Followers in leadership theory: Fiction, fantasy and illusion. Leadership, 14(1), 3-24. Doi: https://doi.org/10.1177/1742715015621372

Gervais, B. (2015) Incivility Online: Affective Behavioral Reaction to Uncivil Political Posts in a Web-based Experiment. Journal of Information Technology and Politics. 12(2), pp. 167-185.

Kollock, P. (1998) Design Principles for Online Communities. Harvard Conference on the Internet and Society, 15(5), 58-60.

Kim, A. (2003) Nine Principles of Community Design. Available: https://people.apache.org/ jim/ NewArchitect/webtech/1998/01/kim/index.html [Accessed: January 21, 2020]

Lerman, K. and Galstyan, A. (2008) Analysis of social voting patterns on Digg. Proc. WOSN, ACM Press, 7-12.

Maity, S.K., Kharb, A., Mukherjee, A. (2017). Language Use Matters: Analysis of the Linguistic Structure of Question Texts Can Characterize Answerability in Quora. Proceedings of the Eleventh International AAAI Conference on Web and Social Media. Available: https://www.aaai.org/ocs/index.php/ICWSM/ICWSM17/paper/view/15647/14866

Mamykina, L., Manoim, B., Mittal, M., Hripcsak, G., Hartmann, B. Design lessons from the fastest Q\&A site in the West. Proc. CHI 2011, ACM Press, 2857-2866.

Meindl, JR (1995) The romance of leadership as a follower-centric theory: A social constructionist approach. The Leadership Quarterly 6: 329-341.

Nowell, L. S., Norris, J. M., White, D. E., and Moules, N. J. (2017) Thematic Analysis: Striving to Meet the Trustworthiness Criteria. International Journal of Qualitative Methods. https://doi.org/10.1177/1609406917733847

Oldenberg, R. (1999). The Great Good Place. Available: https://www.mnsSu.edu/voices/greatgoodplace.pdf

Patil, S., Lee, K. (2016) Detecting experts on Quora: by their activity, quality of answers, linguistic characteristics and temporal behaviors. Soc. Netw. Anal. Min. 6, 5. https://doi.org/10.1007/s13278-015-0313-x

Paul, S.A., Hong, L., Chi. E.H. (2012). Who is authoritative? Understanding reputation mechanisms in quora. Available: https://www.researchgate.net/publication/224053714

Peh, J. (2017) How many questions have been asked on Quora. Available: https://www.quora.com/How-many-questionshave-been-asked-on-Quora-1/answers/19190364

Quora (2020) Why Quora Exist. Available https://www.quora.com/about

Shamir, B, Pillai, R, Bligh, M (2007) Follower-Centered Perspectives on Leadership: A Tribute to the Memory of James R. Meindl, Greenwich, CT: Information Age Publishing.

Strauss, A., and Corbin, J. (1990) Basics of Qualitative Research: Grounded Theory Procedures and Techniques. Sage Publications, Newbury Park, CA, USA.

Tolstikov-Mast, Yulia. (2016) Global Followership: The Launch of the Scholarly Journey. Advances in Global Leadership, pp. 107-150. Doi: 10.1108/S1535-120320160000009013.

Vollstedt, M., Rezat, S. (2019) An Introduction to Grounded Theory with a Special Focus on Axial Coding and the Coding Paradigm. Compendium for Early Career Researchers in Mathematics Education,13, pp. 81- 100. Doi: https://doi.org/10.1007/978-3-030-15636-7_4

Wang, G., Gill, K., Mohanlal, M., Zheng, H., and Zhao, B.Y. (2013). Wisdom in the Social Crowd: an Analysis of Quora. IW3C2. ACM 978-1-4503-2035-1/13/05.

Warwick Institute for Employment Research (IER) (2014) Analyzing Interview Data. University of Warwick. Available: https://warwick.ac.uk/fac/cross_fac/esrcdtc/researchandtraining/ct201314/quals/analysing_interview_data_2014_wk3 _for_web.pdf [Accessed: March 23 2020].

Weeks, B.E., Ardèvol-Abreu, A., de Zúñiga, G.H. (2017) Online Influence? Social Media Use, Opinion Leadership, and Persuasion. International Journal of Public Opinion Research, 29(2), pp. 214-239.https://doi.org/10.1093/ijpor/edv050

Zhu, Haiyi \& Kraut, Robert \& Kittur, Aniket. (2012) Effectiveness of Shared Leadership in Online Communities. Proceedings of the ACM Conference on Computer Supported Cooperative Work, CSCW. 407-416. 10.1145/2145204.2145269. 